\section{Virchow, Rudolf Ludwig Karl}

\author{
A. M. Gressner ${ }^{1}$ und O. A. Gressner ${ }^{2}$ \\ ${ }^{1}$ Labor Dr. Wisplinghoff Berlin, Berlin, Deutschland \\ ${ }^{2}$ Labor Dr. Wisplinghoff Köln, Köln, Deutschland
}

\section{Synonym(e) Biographie}

Lebensdaten Deutscher Arzt, geboren am 13.Oktober 1821 in Schivelbein (Hinterpommern), gestorben am 5. September 1902 in Berlin.

Verdienste Studium der Medizin an der Militärärztlichen Akademie (Pépinière) in Berlin. Professor für pathologische Anatomie in Würzburg (1849-1856) und Berlin (ab 1856), entwickelte die Zellenlehre und begründete die Zellularpathologie. Stellte den Grundsatz auf, dass die Zelle das letzte und kleinste Formelement aller lebendigen Erscheinungen im
Gesunden wie im Kranken ist. Krankheit wird damit auf Zellveränderungen zurückgeführt. Mit der Zellularpathologie wurden die herrschenden humoral- und neuropathologischen sowie vitalistischen Theorien überwunden und Krankheiten als Veränderungen physikalisch-chemischer Prozesse der Zellen aufgefasst. Zusätzlich viele Untersuchungen zur Thrombose und Embolie, zu malignen Tumoren, zur Anthropologie, Ethnologie und prähistorische Forschungen. Politische Aktivitäten als Abgeordneter im preußischen Landtag und als Stadtverordneter mit Schwerpunkten im kommunalen Gesundheitswesen, Schulwesen, Museumsaufbau u. a. Virchow gehört zu den universellsten Gelehrten der Geschichte.

\section{Literatur}

Goschler C (2003) Rudolf Virchow - Mediziner - Anthropologe - Politiker. Böhlau Verlag, Köln 